\title{
Smiling as negative feedback affects social decision-making and its neural underpinnings
}

\author{
Martin Weiß $^{1}$ (D) $\cdot$ Patrick Mussel $^{2} \cdot$ Johannes Hewig $^{1}$ \\ Published online: 3 January 2020 \\ (C) The Psychonomic Society, Inc. 2020
}

\begin{abstract}
A crucial aspect of social decision-making is the ability to learn from the outcomes of preceding decisions. In particular, learning might be influenced by the expectedness of feedback and its valence. Expectedness has largely been operationalized as the frequency of stimulus occurrence and not in terms of its social context. Therefore, we investigated the influence of socially unexpected feedback, i.e., smiling upon adverse events, on behavioral and neural responses. We used a modified version of the ultimatum game, a commonly used paradigm for economic decision-making, by implementing different proposer identities with a distinct reaction pattern towards accepted and rejected monetary offers. We could show that an identity, who reacted with a smile towards rejected offers, evoked lower acceptance rates compared to identities, who reward acceptance with a smile. Electrophysiological correlates indicate N170 effects for emotional identities compared to a neutral control identity. Regarding FRN and P3 brain potentials, we detected a particular function of the smiling face when used as a socially unexpected, negative feedback stimulus. Hence, individuals seek an unexpected smile despite the associated monetary loss, which is accompanied by distinct neural patterns.
\end{abstract}

Keywords Decision-making $\cdot$ Emotional feedback $\cdot$ Ultimatum game $\cdot$ Unexpectedness

\section{Introduction}

Humans often learn from their experience when it comes to social decision-making. There is a consensus that we learn and then adjust future action selection based on the evaluation of consequences and influences of our own behavior toward others (Huang \& Yu, 2014; Maier \& Steinhauser, 2013). However, the behavioral and neural correlates of unexpected positive feedback toward punishing behavior have not been in the focus of research yet.

The neural underpinnings of feedback evaluation have gained increasing attention in the past. Research using

Electronic supplementary material The online version of this article (https://doi.org/10.3758/s13415-019-00759-3) contains supplementary material, which is available to authorized users.

Martin Weiß

martin.weiss@uni-wuerzburg.de

1 Department of Psychology I, Personality Psychology, and Psychological Diagnostics, Julius-Maximilians-Universität Würzburg, Marcusstraße 9-11, 97070 Würzburg, Germany

2 Division Personality Psychology and Psychological Assessment, Freie Universität Berlin, Berlin, Germany event-related potentials (ERPs) has identified a brain component, the feedback-related negativity (FRN; Miltner, Braun, \& Coles, 1997), which is sensitive to the valence of feedback. Holroyd and Coles (2002) and Holroyd, Pakzad-Vaezi, and Krigolson (2008) stated that the FRN indicates activation of a reinforcement learning system, which evaluates the outcome of a distinct decision to direct reward-oriented behavior. The FRN is generated when a negative signal for reinforcement learning is conveyed to the anterior cingulate cortex (ACC) via the mesencephalic dopamine learning system. Holroyd and Coles (2002) suggest that the system classifies feedback into events that are better or worse than expected and computes the difference between outcome and expectation as a reward prediction error (Holroyd et al., 2008). Alternatively, Alexander and Brown (2011) stated in their theory on the prediction of response-outcome that the ACC prognoses possible outcomes of behavior and indicates unexpected nonoccurrence of these outcomes. Thereby, the unexpectedness relates to both negative and positive outcomes. In the present study, we used positive feedback (i.e., a smiling facial expression) toward an adverse event (i.e., rejection of an ultimatum game offer, which means monetary nonreward). Hence, we investigated neural responses of feedback that in terms of frequency is fully expectable but in terms of social behavior might be unexpected. 
In order to study (socially) unexpected facial expressions, we needed a controllable social environment, as in the ultimatum game (UG; Güth, Schmittberger, \& Schwarze, 1982), which is a commonly used economic game for investigating human decision-making. In this task, one party is acting as a "proposer" and must divide a certain amount of money to share with the "responder." The responder subsequently accepts or rejects the offered amount of money. If the responder accepts an offer, the money is divided as proposed. Otherwise, no money will be distributed. For most people, offering 5 cents out of 10 would be considered a fair offer, whereas 1 cent would be considered unfair. According to rational choice theory (Morgenstern \& Von Neumann, 1953), a rational proposer would offer the smallest possible amount to maximize his gains, and a rational responder would accept any offer, because receiving any money is better than gaining nothing. In contrast to rational choice theory, empirical findings show that offers below $20 \%$ of the money are rejected in $50 \%$ of all cases; and proposers offer approximately $40-45 \%$ of the money to the responder (Levine, 1998).

Recently, Mussel, Hewig, and Weiß (2018) and Weiß, Mussel, and Hewig (2019b) modified the UG by implementing different proposer identities, who reacted with distinct patterns of socioemotional feedback after participants accepted or rejected UG offers. On a behavioral level, systematic emotional feedback influenced decision-making in the task. Participants showed higher acceptance rates if the coplayer consistently smiled upon acceptance compared to proposers showing neutral facial expressions. Building upon Mussel et al. (2018), we developed an additional modification of the UG, which allowed us to investigate the behavioral and neural consequences of socially unexpected positive feedback. A particular social framing could turn a stimulus that is usually associated with reward (i.e., a smile) into a stimulus covering negative content and thus lead to increased rejection rates. As an example of positive facial expressions as compensation for negative emotions, Ansfield (2007) showed that smiling when feeling distressed may serve a function in self-regulation. Otherwise, these smiles go along with negative social consequences.

In the present study, by realizing the socially unexpected smile, we also wanted to solve a methodological issue concerning unequally distributed feedback stimuli in preceding studies. Previous publications on emotional feedback (Mussel et al., 2018; Weiß, Gutzeit, Rodrigues, Mussel, \& Hewig, 2019a; Weiß et al., 2019b) worked with different identities, who reacted with emotional expressions to accepted and rejected UG offers. However, among these identities the neutral facial expression was overrepresented (Mussel et al., 2018: in $62.5 \%$ of the conditions, feedback was neutral). The actual frequencies depend on the decisions of the participants, which we cannot control. Only under the assumption of equally frequent acceptance and rejection is the probability now equally distributed for the different emotional facial expressions (50\% neutral, $50 \%$ smiling). This is an advantage over previous studies. Regarding electrophysiology, we wanted to address the effects of social decision-making and emotional faces on N170, FRN, and P3 brain potentials. When watching human facial expressions compared with nonfacial stimuli, the N170 brain potential is larger, indicating a facesensitive function of N170. Therefore, N170 is an established correlate of the visual processing of human faces (Bentin, Allison, Puce, Perez, \& McCarthy, 1996; Eimer, 2000; Rossion et al., 2000). Mussel et al. (2018) reported that the N170 reflected the processing of basic facial features since emotional faces elicited larger amplitudes for the presentation of faces without context and as feedback stimuli in the UG. In conclusion, we predict a valence effect of smiling faces over neutral faces both when presented context-free and as feedback stimulus toward the acceptance or rejection of an UG offer. Next, we addressed the FRN as an indicator for positive and negative feedback processing (Miltner et al., 1997). In order to investigate the neural underpinnings of social feedback, facial expressions have been linked to FRN brain responses in several studies. Pfabigan, Alexopoulos, Bauer, Lamm, and Sailer (2011) reported that FRN was particularly large after negative and unexpected social feedback in a gambling task. With regard to the ultimatum game, Mussel, Hewig, Allen, Coles, and Miltner (2014) showed that a smiling compared with a neutral face preceding the offer not only led to higher acceptance rates but also to smaller FRN amplitudes. In contrast, Schreiner, Alexopoulos, Pfabigan, and Sailer (2010) showed that the FRN would be more pronounced for smiling proposers offering an unfair amount of money than for angry proposers making the same unfair offer. Osinsky, Mussel, Ohrlein, and Hewig (2014) found that the face of a proposer who always makes unfair offers provokes a similar FRN brain response as to the offer itself. In addition, Mussel et al. (2018) found that the desired feedback expressions of an interaction partner (i.e., anger feedback after rejection of an unfair offer) led to a decrease in FRN amplitudes compared with looking at the anger face without context. Accordingly, some studies showed increased FRN for unexpected and/or negative social feedback, whereas others showed decreased FRN for positive and/or expected social feedback. Thus, we investigated the FRN in the context of socially unexpected positive feedback in the present study. We hypothesized that FRN amplitudes are smaller for unexpected positive feedback (i.e., following the rejection of an offer) in terms of social content compared with neutral feedback following the acceptance of an offer. This hypothesis is based on a valence account of FRN (Holroyd et al., 2008) rather than on an unexpectedness account of FRN (Alexander \& Brown, 2011). Finally, the P3 component was analyzed to investigate attentional processing and subjective importance (Ito, Larsen, Smith, \& Cacioppo, 1998; Johnson, 
1988; Polich, 2007) of UG offers and emotional feedback. Several studies (Hajcak, Holroyd, Moser, \& Simons, 2005; Hajcak, Moser, Holroyd, \& Simons, 2007; Johnson \& Donchin, 1985) reported a valence-related modulation of P3 in decision tasks, indicating a larger positivity for positive compared with negative feedback. In the context of the UG, the P3 component was pronounced for fair compared with unfair offers (Qu, Wang, \& Huang, 2013; Wu, Hu, van Dijk, Leliveld, \& Zhou, 2012). Ma, Hu, Jiang, and Meng (2015) further indicated that this P3 effect was modulated by a proposer's attractiveness. Whereas for attractive proposers no P3 difference regarding the fairness of an offer could be reported, unattractive proposers evoked larger P3 brain potentials for fair compared with unfair offers. In conclusion, Ma et al. (2015) showed that faces could have a meaningful impact on P3 amplitudes in the context of the UG. Moreover, Bellebaum, Kobza, Thiele, and Daum (2011) reported that P3 amplitudes were more prominent when participants gained a reward compared with gaining nothing at all. Regarding emotional feedback, we assume that P3 amplitudes will be heightened especially for the unexpected smile following the rejection of an offer. Although implemented as negative feedback, we believe that its positive valence might elicit larger P3 amplitudes compared with neutral feedback following accepted offers.

On the behavioral level, we expect that the identity who unexpectedly reacts with a smile toward the rejection of an offer will elicit lower acceptance rates compared with identities that reward acceptance with a smile.

The present study was designed to clarify whether unexpected content-related feedback would affect human decisionmaking. Therefore, we used a modified version of the ultimatum game with different proposers, who provide emotional feedback toward acceptance or rejection of an offer. Considering that in previous research expectedness was operationalized by the frequency of stimulus occurrence, the social context was used to investigate the influence of unexpected feedback. ERPs were recorded to analyze the effects of smiling versus neutral faces across a mere presentation task and when used as feedback stimuli within different proposer identities. To the best of our knowledge, this is the first study to investigate the social influence of unexpected positive feedback in a decision-making task.

\section{Methods}

\section{Ethical statement}

The study was performed in accordance with the recommendations of Ethical Guidelines, The Association of German Professional Psychologists (Berufsethische Richtlinien, Berufsverband Deutscher Psychologinnen und
Psychologen). All subjects gave written, informed consent in accordance with the Declaration of Helsinki before they participated in the experiment. During the experiment, a cover story was used, but they were told about this deception as soon as the task was over, which is common practice in psychological experiments.

\section{Participants}

A sample size of 51 was estimated for a medium effect of $\eta_{p}^{2}=$ 0.06, $\alpha=0.05$, and $\beta=0.95$ using $G^{*}$ Power (Faul, Erdfelder, Lang, \& Buchner, 2007). The determination was a based on a conservative estimation of the behavioral influence of different proposer identities on acceptance rates in the ultimatum game (Mussel et al., 2018; Weiß et al., 2019b). Finally, 56 subjects were recruited (37 females; mean age 28.53 years, $S D=9.96$, range 19-62), who participated for monetary compensation of $15 €$. All participants gave written, informed consent and were debriefed after the end of the experiment.

\section{Experimental procedure}

For the present study, we selected five male and five female persons from the Radboud Faces database (Langner et al., 2010), each with two facial emotional expressions: happy and neutral. The first task (Figure 1) was a control task to examine context-free neural responses to emotional facial expressions. Thus, the participants were asked to look at the pictures without providing any action. For each participant, one male and one female person were randomly chosen and subsequently presented 25 times in random order (100 trials in total). Additionally, we conducted a manipulation check on the selected stimuli to evaluate their arousal and valence on a seven-point scale.

In the second task, participants played ten rounds of a modified ultimatum game in the role of the proposer. Primarily, the purpose of this task was to facilitate understanding and enhance the plausibility of the cover story for the third task (see below). In each round, the participants could divide 10 cents into two shares: one for her/himself and one for the responder. There were six predefined shares, ranging from 0 to 5 cents. Irrespective of their choice, the participants subsequently received feedback that their offer was either accepted or rejected (in 50\% of the cases). Finally, participants had the opportunity to send a picture to the other player to express how they felt about his or her decision. For this purpose, the two emotional pictures from Task 1 were presented, and female participants could select a happy or a neutral picture of the female person and vice versa.

In the third task, participants played eight blocks (with 48 trials each) of the modified ultimatum game in the role of the responder. All participants were informed that the offers were 
(a)

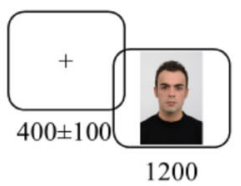

(c)

(d)

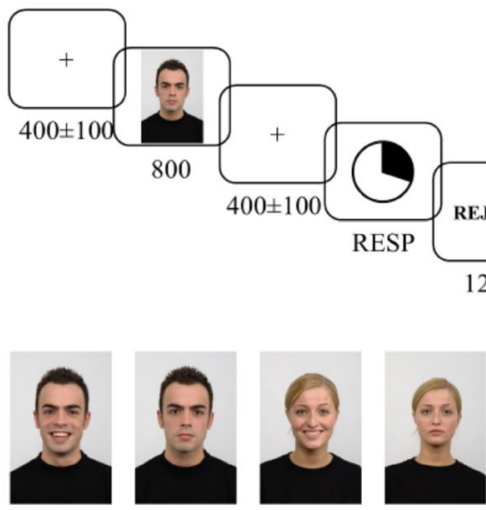

(b)

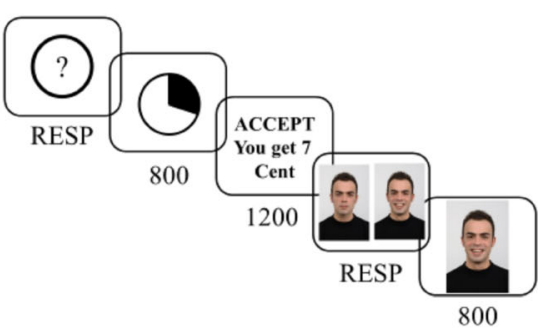

Fig. 1 (a-c) Task timeline for the three paradigms. The numbers indicate presentation time in milliseconds. (d) Example pictures for smiling and neutral facial expressions (Langner et al., 2010).

made by other participants who conducted the experiment on an earlier occasion and that both parties would be paid depending on the decisions made by the participant. Furthermore, we stated that we took a picture of each participant for the subsequent participant. In truth, the UG offers were predefined, as outlined below. Each trial began with a picture of either a male or female person with a neutral facial expression representing the proposer of the upcoming offer. The picture was taken from the pool of the eight persons who were not chosen in Task 1 . Next, the offer made by this proposer was shown, representing a split of 10 cents, ranging from 0 to 5 cents for the responder. The offer was presented as a pie chart (e.g., 2/8 for the responder/proposer). During a block, each of the eight persons proposed each of the six possible offers exactly once. The order of the 48 offers was randomized for each participant. Afterward, the participants decided to either accept or reject the offer by pressing the left (acceptance) or right (rejection) arrow button. Their decision was shown for 1,200 ms along with the gained money (e.g., “Accept! You get 3 Cents!'). Finally, a picture with varying facial expression of the proposer was shown, indicating how he or she felt in answer to the decision of the participant. Thereby, each of the eight persons behaved in one of four particular ways, which we call the identity of this person. The happy identity reacted with a smile if an offer was accepted and a neutral expression if the offer was rejected. The neutral identity reacted with a neutral facial expression, irrespective of the decision of the responder. The content identity reacted with a smile, irrespective of the decision of the responder. Finally, the unexpected identity reacted with a neutral facial expression if the offer was accepted and a smile if the offer was rejected. For each participant, one male and one female person was randomly assigned to one of the four feedback patterns resulting in eight different identities (4 identities represented each by males and females). All stimuli were presented on a 17" screen with a grey background. Stimulus presentation and response recordings were controlled by PsychoPy 1.83 (Peirce, 2008). During the task, participants were seated in a comfortable chair with a distance of $70 \mathrm{~cm}$ between the head and the screen. Each of the pictures was 10$\mathrm{cm}$ high and $6.65-\mathrm{cm}$ wide, resulting in a visual angle of about $14.2^{\circ} \times 9.5^{\circ}$. The pie charts had a diameter of $2.5 \mathrm{~cm}\left(3.6^{\circ}\right.$ visual angle).

\section{EEG recording and quantification}

The EEG was measured by $\mathrm{Ag} / \mathrm{AgCl}$-electrodes located in an electrode cap in 32 scalp positions according to the international 10-20 system: FP1, FP2, Fz, FCz, F3, F4, F7, F8, F9, F10, FC1, FC2, FC5, FC6, CZ, C3, C4, T7, T8, CP1, CP2, TP9, TP10, P3, P4, P7, P8, P09, PO10, O1, and O2. An additional electrode to register eye movements and blinks, called electrooculography (EOG), was put below the left eye. All electrode impedances were kept below $5 \mathrm{kOhm}$ for the EEG, and data were referenced online to the vertex $(\mathrm{Cz})$. Data were recorded with a sampling rate of $500 \mathrm{~Hz}$ and a high cutoff filter of $80 \mathrm{~Hz}$ with BrainVision BrainAMP Standard (Brain Products GmbH, Gilching, Germany) and the respective BrainVision Recorder software. For the processing of the collected EEG data, we used MATLAB (MathWorks, Natick, MA) and EEGLAB (Delorme \& Makeig, 2004). Initially, we detected inappropriate channels for each participant and interpolated them. We segmented epochs from $-500 \mathrm{~ms}$ to 1,000 ms around the target markers. For baseline correction, 
we assessed a window of $-200 \mathrm{~ms}$ to $0 \mathrm{~ms}$. Regarding artifact rejection, we used the criterion of $\mathrm{z}$-value $>4$ for the amplitude and kurtosis of the signal. Before applying independent component analysis (ICA) according to Delorme, Sejnowski, and Makeig (2007), we used a band-pass filter from 1 to $40 \mathrm{~Hz}$. Afterward, we applied the extensions ADJUST (Mognon, Jovicich, Bruzzone, \& Buiatti, 2011) and MARA (Winkler, Haufe, \& Tangermann, 2011), which operate with SASICA software (Chaumon, Bishop, \& Busch, 2015) to choose ICA components for artifact rejection. Offline reference was transformed to current source density like in other ERP studies before (Kayser et al., 2010; Milne, 2011; Weiß et al., 2019a), and data were filtered with a $20-\mathrm{Hz}$ low pass filter. Statistical calculations were conducted for each participant for each condition $\pm 20 \mathrm{~ms}$ around the positive or negative peak, respectively. N170 latency was quantified as the negative peak between 130 and $210 \mathrm{~ms}$ (Johnston, Molyneux, \& Young, 2014; Mussel et al., 2018; Weiß et al., 2019a) pooled at electrodes P7 and P8. Latencies for FRN were calculated as the negative peak between 200 and $400 \mathrm{~ms}$ at electrode site $\mathrm{FCz}$, where their maxima would typically occur (Burle, Roger, Allain, Vidal, \& Hasbroucq, 2008; Gehring \& Willoughby, 2002; Holroyd \& Coles, 2002). The P3 component was calculated as the positive peak between 250 and $400 \mathrm{~ms}$ at $\mathrm{Pz}$ electrode site, as illustrated in Figure 2.

\section{Statistical analyses}

Data were analyzed using repeated-measures analyses of variance (ANOVA) in SPSS software (IBM, Armonk, NY). For all ANOVA reported in the following paragraph, we assumed $\alpha=0.05$. In case of violation of sphericity assumption, epsilon $(\varepsilon)$ and corrected $p$ values are reported. We used Hyunh-Feldt adjustment factors for degrees of freedom, apart from when $\varepsilon$ $<0.75$. In this case, we adjusted the degrees of freedom, according to Greenhouse-Geisser. When we found a statistically significant result for any ANOVA, we computed Bonferroniadjusted pairwise comparisons for further investigation of the effects.
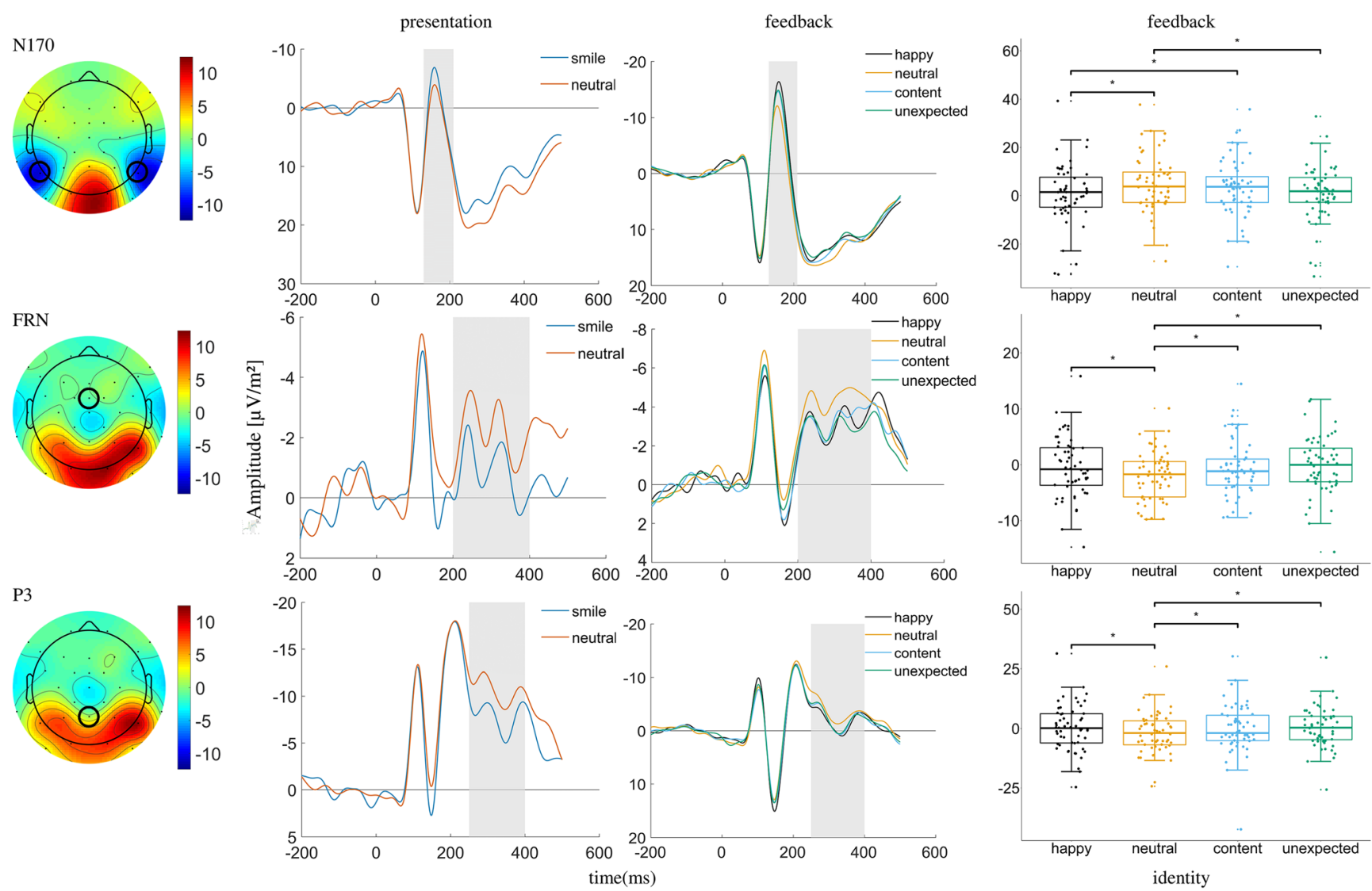

Fig. 2 Grand averages according to the presentation task and emotional feedback. The first row contains results averaged across electrodes P7 and P8 (relevant for N170), the second row results for electrode FCz (relevant for FRN), and the third row for electrode site $\mathrm{Pz}$ (relevant for P3). In the first column, grand averages regarding the presentation task are displayed. In the second column, grand averages for the main effect of

identity in the feedback condition are depicted. Finally, in the third column, individual data for the main effect of identity are displayed with boxplots. The whiskers have the length of 1.5 times the interquartile range. The grey areas highlight the search windows for peak detection. The topographic maps are also based on these time windows and aggregated across stimuli. $* p \leq 0.05$ 
The manipulation check regarding the valence and arousal of the stimuli was analyzed with a one-factorial repeated-measures ANOVA with the factor emotion (two levels: smiling and neutral). In the mere presentation task, N170, FRN, and P3 brain potentials were investigated with a one-factorial ANOVA with the factor emotion (2 levels: smiling, neutral). Behavioral results regarding acceptance rates were obtained by investigating the factors identity (4 levels: happy, neutral, content, unexpected), block (8 levels: $1-8)$, and offer (6 levels: $0-5$ cents). Emotional feedback was investigated with a two-factorial, repeated-measure ANOVA with the factors identity (4 levels: happy, neutral, content, unexpected) and decision (2 levels: acceptance, rejection).

\section{Results}

\section{Manipulation check}

Smiling facial expressions obtained higher valence ratings compared with neutral ones, $F(1,53)=359.01, p<0.001$, $\omega_{\mathrm{p}}^{2}=0.866$. Ratings regarding arousal also depended on the emotional valence of the stimulus, $F(1,53)=38.60, p<0.001$, $\omega_{\mathrm{p}}^{2}=0.406$, because smiling faces were rated higher in arousal compared with neutral facial expressions.

Furthermore, we conducted an explorative analysis on the feedback selection in task 2 , where participants acted as proposers 10 round to validate the concept of unexpected positive social feedback. A chi-square test of independence was performed to examine the relationship between acceptance/ rejection and feedback selection. The relation between these variables was significant, $X^{2}(1, N=56)=374.9, p<0.001$. Positive feedback was selected significantly more often than neutral feedback after acceptance, and neutral feedback was selected significantly more often than positive feedback after rejection.

\section{Behavioral results}

Regarding behavior, we found a significant effect of identity, $F(3,165)=7.69, p<0.001, \omega_{\mathrm{p}}^{2}=0.106, \varepsilon=0.546$, and offer, $F(5,275)=151.80, p<0.001, \omega_{\mathrm{p}}^{2}=0.728, \varepsilon=0.545$. Furthermore, there was a significant interaction of identity and block, $F(21,1155)=2.37, p=0.014, \omega_{\mathrm{p}}^{2}=0.023, \varepsilon=$ 0.407 . The unexpected identity obtained significantly less accepted offers compared with the happy identity $(p=0.014)$ and the content one $(p=0.045)$ but not compared with the neutral identity $(p=0.666)$. Furthermore, the acceptance rates for the happy $(p=0.002)$ and the content identity $(p=0.031)$ were significantly higher compared with the neutral identity, whereas happy and content identities did not differ $(p=1$; Figure 3 ). The comparison of the six offer sizes indicated higher acceptance rates for higher offers. All offers differed significantly (all values of $p<0.001$ ), apart from 4 cents and 5 cents $(p=0.240)$. The significant interaction of identity and block is displayed in Figure 4. In the first two blocks, acceptance rates did not differ significantly (all values of $p \geq 0.715$ ). Beginning in the third block, the main effect of identity was mostly stable across the remaining five blocks corroborating the above findings. We found no significant effect for block, $F(7,385)=0.73, p=0.572, \varepsilon=0.585$; the interaction between identity and offer, $F(15,825)=1.59, p=0.124, \varepsilon=0.534$; the interaction between block and offer, $F(35,1925)=1.09, p=$ $0.354, \varepsilon=0.419$; and the three-way interaction of identity, block and offer, $F(105,5775)=0.82, p=0.718, \varepsilon=0.239$.

\section{Electrophysiological results ${ }^{1}$}

In the presentation task, we detected a significant effect of emotion on N170 brain potentials, $F(1,55)=13.08, p=$ $0.001, \omega_{\mathrm{p}}^{2}=0.174$, indicating that smiling faces evoked larger N170 amplitudes compared with neutral faces. FRN amplitudes $^{2}$ also showed a main effect of emotion, $F(1,55)=$ 9.31, $p=0.003, \omega_{p}^{2}=0.127$. The neutral facial expression elicited larger FRN brain potentials compared to the smiling face. Likewise, the main effect of emotion on P3 brain potentials indicated larger amplitudes when a smiling compared with a neutral face was presented, $F(1,55)=9.31, p=0.003$, $\omega_{\mathrm{p}}^{2}=0.127$.

Regarding emotional feedback and N170 brain potentials, the main effect of identity, $F(3,162)=21.35, p<$ $0.001, \omega_{\mathrm{p}}^{2}=0.268$, was qualified by a significant interaction with decision, $F(3,162)=21.40, p<0.001, \omega_{\mathrm{p}}^{2}=$ $0.269, \varepsilon=0.873$. The neutral identity evoked significantly less negative amplitudes compared with the happy and the unexpected identity (all values of $p \leq 0.045$ ). Furthermore, the happy compared to the content identity evoked more negative amplitudes $(p<0.001)$. No further comparisons yielded significance (all values of $p \geq 0.054$ ). Within the happy identity, the neutral face following rejection evoked more negative amplitudes compared with the smile following acceptance $(p=0.003)$. The neutral identity's facial expressions did not differ $(p=0.335)$. In contrast, the content identity's smile following acceptance evoked significantly more negative N170 brain potentials compared with

\footnotetext{
${ }^{1}$ We also analyzed the effects of the presented offers in the ultimatum game. These results and a short discussion are outlined in the online supporting information.

${ }^{0}$ We observed two peaks in the FRN time-window (Figure 2), and therefore ran separate analyses on the two peaks (time-windows: 200-300 ms and 300$400 \mathrm{~ms}$ ). The results were almost identical to the analyses presented in the Results section. Only within the second time-window, the interaction of identity and decision regarding emotional feedback was significant and not marginally significant, as reported in the Results. The direction of all effects did not deviate from the analyses on the general time-window.
} 


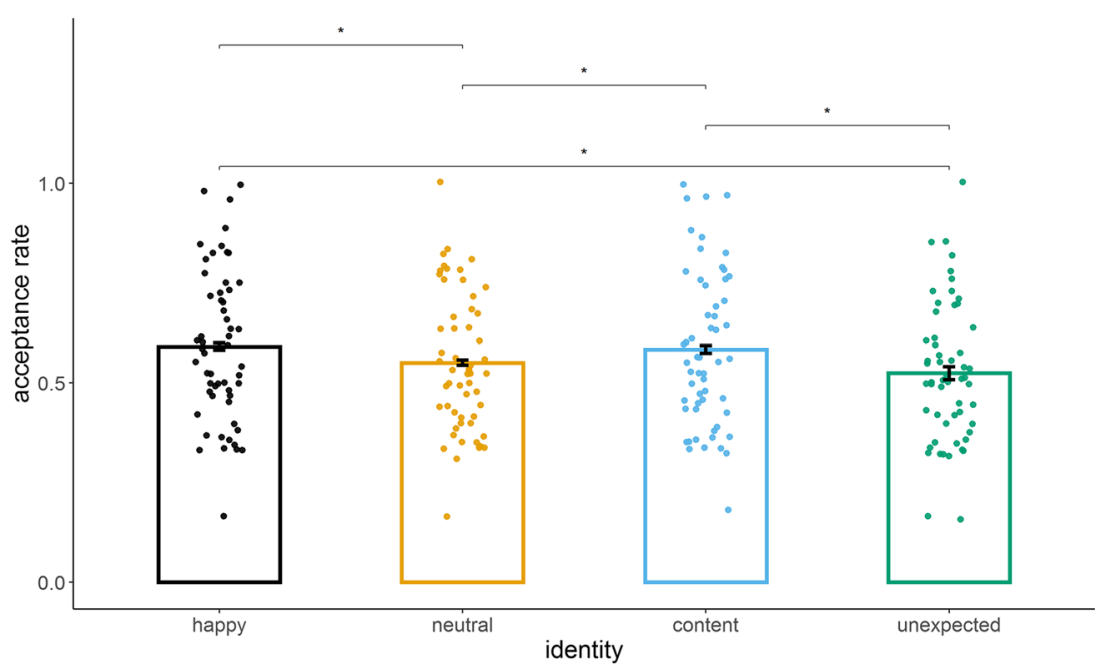

Fig. 3 Average acceptance rates, depending on the identity of the proposer. Each identity reacted with a characteristic pattern of emotional facial expressions towards accepted and rejected offers. Data

the smile after rejection $(p<0.001)$. Regarding the unexpected identity, the smile following rejection evoked more negative amplitudes $(p<0.001)$ compared to the neutral face after acceptance. For the factor decision no effect could be reported, $F(1,54)=1.72, p=0.194$.

Next, we found a significant effect of identity on FRN brain potentials, $F(3,162)=4.62, p=0.004, \omega_{\mathrm{p}}^{2}=0.061$. The neutral identity evoked significantly more negative amplitudes compared to all other identities (all values of $p \leq 0.039$ ). We examined a marginally significant interaction between identity and decision, $F(3,162)=2.59, p=0.055, \omega_{\mathrm{p}}^{2}=0.027$. Within the unexpected identity, the neutral face following acceptance elicited more negative FRN brain potentials than the smiling towards rejection ( $p=0.013$ ), as illustrated in Figure 5. No further comparisons yielded significance (all values of $p \geq$ points represent the mean acceptance rate of each subject per identity. Error bars indicate standard error of the mean. * $p \leq 0.05$

0.271). Concerning the factor decision, no effect was found, $F(1,54)=1.66, p=0.203$.

Finally, the main effect of identity on $\mathrm{P} 3$ brain potentials, $F(3,162)=5.39, p=0.001, \omega_{\mathrm{p}}^{2}=0.073, \varepsilon=0.896$, was qualified by a significant interaction between identity and decision, $F(3,162)=5.39, p=0.001, \omega_{\mathrm{p}}^{2}=0.073$. The neutral control identity elicited significantly less positive $\mathrm{P} 3$ amplitudes compared with all other identities (all values of $p \geq 0.021$ ). Within the unexpected identity, rejection (i.e., a smile) evoked more positive P3 amplitudes compared with acceptance $(p=0.001$; Figure 5). For the content identity, the smile toward acceptance elicited more positive $\mathrm{P} 3$ amplitudes compared with the smile following rejection $(p=0.032)$, whereas all other identities showed no significant differences (all values of $p \geq 0.278$ ). Decision did not show any effect, $F(1,54)=0.93, p=0.339$.

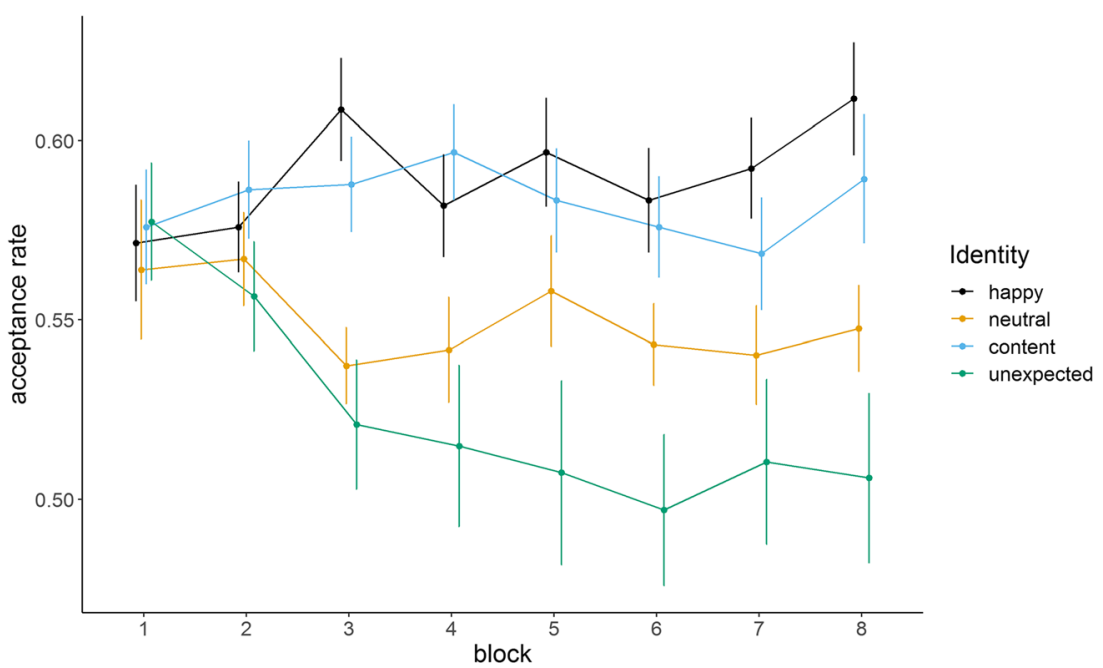

Fig. 4 Average acceptance rates, depending on the block in the task and the identity of the proposer. The plot indicates learning curves as a significant interaction of block and identity. Error bars indicate standard error of the mean. 
N170

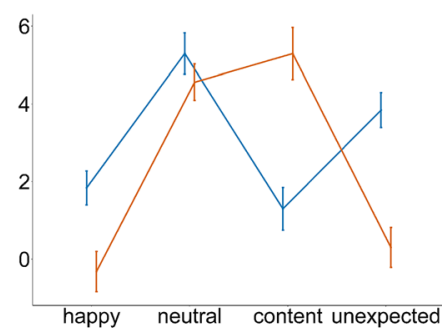

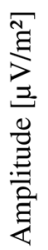

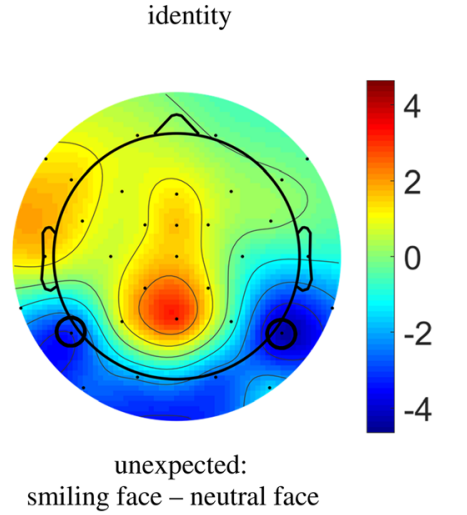

FRN
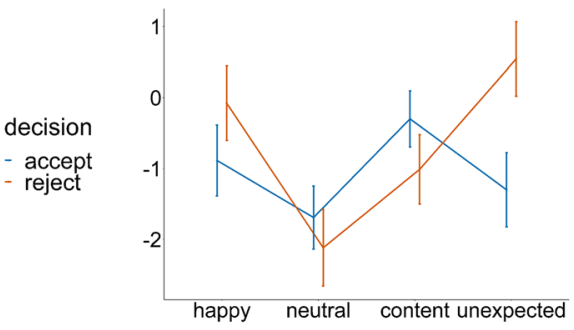

identity

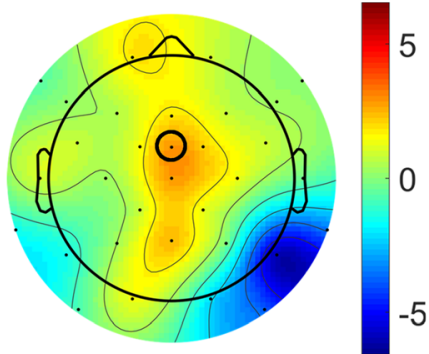

unexpected: smiling face - neutral face
P3

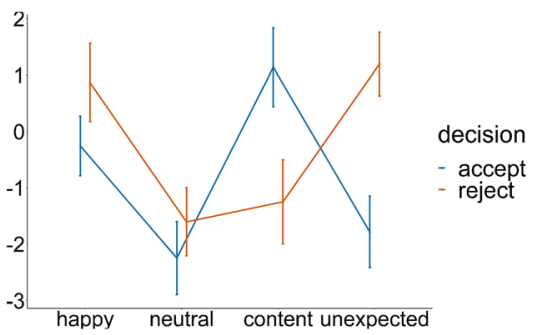

identity

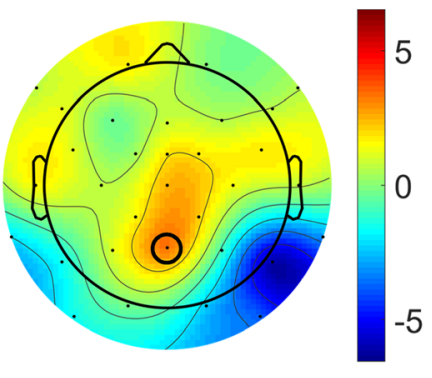

unexpected: smiling face - neutral face
Fig. 5 Interaction of identity and decision in the feedback task. The first row indicates the two-way interaction of identity and decision for N170, FRN, and P3 brain potentials, respectively. The second row shows difference topographic maps for the unexpected identity. The unexpected identity reacted with a smile toward rejection and a neutral face toward acceptance. In the first column, rejection-acceptance at the minima for the

\section{Discussion}

We investigated behavioral and neural responses to different proposer identities in a modified ultimatum game with emotional feedback. As a novel contribution, the present study focused on how socially unexpected feedback alters acceptance rates and underlying neurophysiological correlates. Thereby, we were able to show the influence of emotional feedback toward behavior in the ultimatum game, as initially reported by Mussel et al. (2018). In the present study, participants more often accepted offers coming from proposers who smile toward accepted offers and who smile independent of the responder's decision. In contrast, acceptance rates were lower for the neutral identity and the unexpected identity who smiled upon rejection.

Descriptively, the unexpected identity reached the lowest acceptance rate (52\%) followed by the neutral (55\%), the content $(58 \%)$, and the happy identity (59\%). According to our assumptions, we were able to create an identity that altered behavior, although by positive feedback toward rejection. The unexpected feedback may be linked conceptually to personality traits, such as cynicism. Selfish and reckless attributes of cynics (Dean, Brandes, \& Dharwadkar, 1998; Graham \& Graham, 1990) could facilitate anger, sadness, and withdrawal from social interactions (Greenglass \& Julkunen, 1989).
$\mathrm{N} 170$ component $(160 \mathrm{~ms})$ at the $\mathrm{P} 7$ and $\mathrm{P} 8$ electrode sites is indicated. In the second column, rejection-acceptance at the minima for the FRN (300 $\mathrm{ms}$ ) at the $\mathrm{FCz}$ electrode site is indicated, and in the third column rejection-acceptance at the maxima for $\mathrm{P} 3(333 \mathrm{~ms})$ at the $\mathrm{Pz}$ electrode site, respectively

Consequently, lower acceptance rates for the unexpected identity mirrored findings reported by Demerouti, Xanthopoulou, and Bakker (2018), who investigated cynical behavior in an employee-customer relation. The authors reported that cynical employees showed more negative actions and reactions toward customers, which subsequently led to less satisfied customers. A second interpretation might be that a smile after the rejection of an offer merely evokes aggression, which could be intended to signal dominance, or that it might appear simply odd or irritating. Furthermore, for individuals who strive for the reward of a smiling face, the rejection of offers coming from the unexpected identity yielded immediate positive feedback in terms of reinforcement learning. However, seeking reward would lead to a loss of money, which would yield a strong conflict as the reward of the smile goes along with the absence of a monetary reward and vice versa. Finally, the meaning of the smile could vary as a function of offer. As Niedenthal, Mermillod, Maringer, and Hess (2010) state, a human face expressing a smile is the most complex facial expression and therefore may transport very complex messages. Possibly, positive feedback following the rejection of an unfair offer might be interpreted as some sort of apology by the proposer.

People encounter and learn from dyadic social interactions over time. Therefore, we investigated learning curves across 
the blocks for the proposer identities in the ultimatum game. As the interleaved design in the present task was rather complex, the participants needed the first two blocks of trials to get to know the distinct feedback patterns of the proposers. However, after these two initial blocks, the acceptance rates began to diverge. In the intermediate blocks, the identity with the highest acceptance rate switched between the happy and the content identity, both rewarding acceptance with a smile. The neutral and the unexpected identity, both responding with a neutral face to acceptance, steadily received the lowest acceptance rates. Interestingly, the unexpected identity obtained continuously lower acceptance rates over the course of time. Hence, social learning might imply that individuals use (un-)successful interactions to reevaluate the meaning of social cues (Zaki, Kallman, Wimmer, Ochsner, \& Shohamy, 2016).

In the mere presentation task, we found a frequently reported effect of emotional valence on N170 brain potentials (Almeida et al., 2014; Blau, Maurer, Tottenham, \& McCandliss, 2007; Blechert, Sheppes, Di Tella, Williams, \& Gross, 2012; Hinojosa, Mercado, \& Carretié, 2015), as smiling faces elicited more pronounced N170 amplitudes compared with neutral ones. Regarding FRN, we observed that neutral facial expressions compared to smiling ones elicited more negative brain potentials. In line with the results reported by Mussel et al. (2018), the FRN might signal the conflict processing toward neutral faces, because they do not indicate a negative or positive emotion. Furthermore, smiling faces are inherently rewarding as compared to neutral facial expressions. They can be interpreted as feedback stimuli, which are better than expected and thus elicit a reduced FRN or a feedback positivity (Holroyd et al., 2008). Pronounced P3 amplitudes for smiling faces might further indicate the benefits of a smile, because it inherently might be more salient to an individual compared with a possibly unimportant neutral facial expression. At this point, we want to mention that the FRN in both presentation and feedback task is somewhat noisy, which might have occurred due to our relatively short interstimulus interval (for discussion on the influence of interstimulus intervals on P3 amplitudes see Sambeth, Maes, \& Brankačk, 2004). We therefore point to evaluate the FRN results with caution.

Concerning emotional feedback, we reported an effect of valence on N170 brain potentials. Both the content and the unexpected identity evoked more negative deflections compared with the neutral control. However, the interaction revealed that for the happy identity, the neutral face following rejection elicited more negative amplitudes than the smile following acceptance. In contrast, within the unexpected identity, the smile upon rejection compared with the neutral face upon acceptance evoked greater N170 amplitudes. Calvo and Nummenmaa (2016) presume that the differentiation between emotional and nonemotional affective faces is based on arousal. Thus, the N170 component might have indicated arousal toward feedback after a subject rejected an offer preceding the valuation of its emotional valence. Positive and neutral feedback after a monetary reward might not be that arousing as the reward per se. However, feedback after nonreward might add arousal to the monetary nonreward. Because this effect only occurred when stimuli differed within an identity, the relativity of rejection versus acceptance feedback might play a key role in this arousal component. Next, the FRN results followed our predictions. We expected the smallest FRN for unexpected positive feedback after rejecting an (unfair) offer. Two different explanatory approaches are discussed below. First, we will focus on the similarity between the mere presentation task and the feedback effects. Second, we will provide possible interpretations for the expected FRN effect for unexpected positive feedback. The FRN results of social feedback mirrored the findings of the presentation task, because the control identity compared with the other identities evoked more negative FRN brain potentials. In consequence, the absence of emotional feedback seemed to evoke greater conflict or a more negative evaluation compared with the other feedback patterns used in this study, which is in line with previous research reporting enhanced FRN for conflict monitoring (Donkers \& Van Boxtel, 2004). Moreover, the absence of reward within the control identity might have caused pronounced negativity compared with identities that reward either acceptance or rejection with a smile. Bellebaum and Daum (2008) showed that the FRN for expected nonreward in a learning paradigm was significantly larger compared with expected reward. Accepting an offer from the unexpected identity did not lead to a rewarding stimulus compared with rejecting an offer from this proposer (neutral face vs. smiling face). Therefore, the larger FRN amplitudes for acceptance feedback might reflect a conflict about the absence of expected positive feedback. Similar to the N170 results, the relativity of acceptance versus rejection feedback might drive this effect. Whereas for the happy identity the positive feedback upon acceptance and the neutral feedback upon rejection coincide with expectable behavior, the feedback pattern of the unexpected identity contradicts expectations. Alternatively, the smile invited subjects to reject offers, thus leading to a decrease in FRN. Hence, participants could have aimed to provoke the positive feedback of the unexpected identity, which could be the reason for less negative brain potentials toward the intended reaction ( Mussel et al., 2018). Furthermore, individuals underlie an optimism bias (Sharot, 2011). A misprediction of future occurrences (i.e., the assumption that the unexpected identity would sometime also smile upon acceptance) might lead to a reduced processing of the feedback towards an adverse event (i.e., loss of money), as the social feedback was rewarding. Decreased FRN amplitudes for the feedback smile might further indicate that the smile was perceived as a polite apology of the proposer for his or her unfair offer or an acknowledgement of having done 
something wrong. The reward-like neural response to the smile might thus be interpreted as successful costly punishment, i.e., the receiver renounced a financial benefit in order to penalize the proposer for his or her unfair offer, and the emotional facial expression of the proposer signals that the action was successful.

Regarding P3 brain potentials, we found the expected larger amplitudes for rejection feedback (i.e., a smiling face) of the unexpected identity. Similar to the results of the FRN, P3 results are first discussed in relation to the presentation task and then in relation to the feedback evaluation. As in the presentation task, smiling faces as feedback after accepted offers and the smiling face after rejected offers of the unexpected identity showed larger P3 amplitudes than the neutral faces. However, the smiling face after rejection from the content identity deviated from this pattern and showed P3 amplitudes of similar magnitude as the neutral feedback faces. Hence, the context of feedback valence between and within identities seems to alter its neural representation. The increased P3 brain potentials for proposers who reacted emotional with a smile to either acceptance or the smile after rejection from the unexpected identity might indicate a particular strong recognition of these identities. For instance, Calvo and Beltrán (2013) showed that P3 amplitudes for loved faces were larger compared with babies, neutral, famous, and unknown faces. Although not necessarily loved, the affective proposers might be more likeable than the neutral control. Furthermore, the unexpected identity's smile upon rejection compared with the neutral face following acceptance might reflect an even stronger recognition as the smile was socially unexpected.

We briefly address some of the limitations of the present study. First, we did not assess the concrete meaning of the feedback smile following the rejection of an offer. We could have asked the participants to rate each of the identities according to social affective dimensions such as likeability and trustworthiness. Second, a probably confounding factor when interpreting the findings of the present study is that, according to our manipulation check, smiling faces were rated as more arousing, compared to neutral facial expressions. According to Schupp, Markus, Weike, and Hamm (2003), ERPs are sensitive to the arousal of a stimulus, independent of its valence. Furthermore, Bradley, Greenwald, Petry, and Lang (1992) reported that viewing time and the memorization of pictures is also related to higher arousal, indicating enhanced attention and encoding. Hence, an elevated level of motivational and affective attraction by the smiling faces might have produced some artificial effects, because they were physiologically more relevant than the lower arousing neutral faces.

\section{Conclusions}

In the present study, we focused on the emotional influence of an unexpected acting identity who reacted with positive feedback (i.e., a smile) toward an adverse event, i.e., the rejection of an offer in the ultimatum game. The distinctive character of this kind of interaction partner is accentuated by obtaining lower acceptance rates compared with identities that reward acceptance with a smile. Further evidence stems from neural components, as FRN brain potentials indicate heightened negativity for the acceptance feedback coming from the unexpected identity (a neutral face). Additionally, larger P3 brain potentials for the smile provided by the unexpected identity compared to the neutral face following acceptance indicated subjective relevance. To sum up, we were able to show that positive feedback does not necessarily have to be interpreted as such, and by social framing, it could have different meanings, such as a friendly apology.

Acknowledgments The authors thank Rouven Aust, Corinna Birner, and Simeon Schäfer for collecting the data presented in this study.

Funding statement European Union "Individualisierung Digital" project (Fonds 823881) in the Europäischer Fonds für regionale Entwicklung (EFRE).

Neither of the experiments reported in this article was formally preregistered. Neither the data nor the materials have been made available on a permanent third-party archive; requests for the data or materials can be sent via email to the correspondence author at martin.weiss@uniwuerzburg.de.

\section{References}

Alexander, W. H., \& Brown, J. W. (2011). Medial prefrontal cortex as an action-outcome predictor. Nature Neuroscience, 14(10), 1338. doi: https://doi.org/10.1038/nn.2921

Almeida, P. R., Ferreira-Santos, F., Vieira, J. B., Moreira, P. S., Barbosa, F., \& Marques-Teixeira, J. (2014). Dissociable effects of psychopathic traits on cortical and subcortical visual pathways during facial emotion processing: An ERP study on the N 170. Psychophysiology, 51(7), 645-657. doi:https://doi.org/10.1111/psyp.12209

Ansfield, M. E. (2007). Smiling when distressed: When a smile is a frown turned upside down. Personality and Social Psychology Bulletin, 33(6), 763-775. doi:https://doi.org/10.1177/0146167206297398

Bellebaum, C., \& Daum, I. (2008). Learning-related changes in reward expectancy are reflected in the feedback-related negativity. European Journal of Neuroscience, 27(7), 1823-1835. doi:https:// doi.org/10.1111/j.1460-9568.2008.06138.x

Bellebaum, C., Kobza, S., Thiele, S., \& Daum, I. (2011). Processing of expected and unexpected monetary performance outcomes in healthy older subjects. Behavioral Neuroscience, 125(2), 241. doi: https://doi.org/10.1037/a0022536

Bentin, S., Allison, T., Puce, A., Perez, E., \& McCarthy, G. (1996). Electrophysiological studies of face perception in humans. Journal of Cognitive Neuroscience, 8(6), 551-565. doi:https://doi.org/10. 1162/jocn.1996.8.6.551

Blau, V. C., Maurer, U., Tottenham, N., \& McCandliss, B. D. (2007). The face-specific N170 component is modulated by emotional facial expression. Behavioral and Brain Functions, 3(1), 7. doi:https:// doi.org/10.1186/1744-9081-3-7

Blechert, J., Sheppes, G., Di Tella, C., Williams, H., \& Gross, J. J. (2012). See What You Think: Reappraisal Modulates Behavioral and Neural 
Responses to Social Stimuli. Psychological Science, 23(4), 346-353. doi:https://doi.org/10.1177/0956797612438559

Bradley, M. M., Greenwald, M. K., Petry, M. C., \& Lang, P. J. (1992). Remembering pictures: pleasure and arousal in memory. Journal of Experimental Psychology: Learning, Memory, and Cognition, 18(2), 379. doi:https://doi.org/10.1037/0278-7393.18.2.379

Burle, B., Roger, C., Allain, S., Vidal, F., \& Hasbroucq, T. (2008). Error negativity does not reflect conflict: a reappraisal of conflict monitoring and anterior cingulate cortex activity. Journal of Cognitive Neuroscience, 20(9), 1637-1655. doi:https://doi.org/10.1162/jocn. 2008.20110

Calvo, M. G., \& Beltrán, D. (2013). Recognition advantage of happy faces: tracing the neurocognitive processes. Neuropsychologia, 51(11), 2051-2061. doi:https://doi.org/10.1016/j.neuropsychologia. 2013.07.010

Calvo, M. G., \& Nummenmaa, L. (2016). Perceptual and affective mechanisms in facial expression recognition: An integrative review. Cognition and Emotion, 30(6), 1081-1106. doi:https://doi.org/10. 1080/02699931.2015.1049124

Chaumon, M., Bishop, D. V., \& Busch, N. A. (2015). A practical guide to the selection of independent components of the electroencephalogram for artifact correction. Journal of Neuroscience Methods, 250, 47-63. doi:https://doi.org/10.1016/j.jneumeth.2015.02.025

Dean, J. W., Brandes, P., \& Dharwadkar, R. (1998). Organizational cynicism. Academy of Management Review, 23(2), 341-352.

Delorme, A., \& Makeig, S. (2004). EEGLAB: an open source toolbox for analysis of single-trial EEG dynamics including independent component analysis. Journal of Neuroscience Methods, 134(1), 9-21. doi:https://doi.org/10.1016/j.jneumeth.2003.10.009

Delorme, A., Sejnowski, T., \& Makeig, S. (2007). Enhanced detection of artifacts in EEG data using higher-order statistics and independent component analysis. Neuroimage, 34(4), 1443-1449. doi:https://doi. org/10.1016/j.neuroimage.2006.11.004

Demerouti, E., Xanthopoulou, D., \& Bakker, A. B. (2018). How do cynical employees serve their customers? A multi-method study. European Journal of Work and Organizational Psychology, 27(1), 16-27. doi:https://doi.org/10.1080/1359432X.2017.1358165

Donkers, F. C., \& Van Boxtel, G. J. (2004). The N2 in go/no-go tasks reflects conflict monitoring not response inhibition. Brain and Cognition, 56(2), 165-176. doi:https://doi.org/10.1016/j.bandc. 2004.04.005

Eimer, M. (2000). The face-specific N170 component reflects late stages in the structural encoding of faces. Neuroreport, 11(10), 2319-2324. doi:https://doi.org/10.1097/00001756-200007140-00050

Faul, F., Erdfelder, E., Lang, A. G., \& Buchner, A. (2007). G*Power 3: a flexible statistical power analysis program for the social, behavioral, and biomedical sciences. Behavior Research Methods, 39(2), 175191. doi:https://doi.org/10.3758/BF03193146

Gehring, W. J., \& Willoughby, A. R. (2002). The medial frontal cortex and the rapid processing of monetary gains and losses. Science, 295(5563), 2279-2282. doi:https://doi.org/10.1126/science. 1066893

Graham, J. R., \& Graham, J. R. (1990). MMPI-2: Assessing Personality and Psychopathology: Oxford University Press New York.

Greenglass, E. R., \& Julkunen, J. (1989). Construct validity and sex differences in Cook-Medley hostility. Personality and Individual differences, 10(2), 209-218. doi:https://doi.org/10.1016/01918869(89)90206-7

Güth, W., Schmittberger, R., \& Schwarze, B. (1982). An experimental analysis of ultimatum bargaining. Journal of Economic Behavior \& Organization, 3(4), 367-388. doi:https://doi.org/10.1016/01672681(82)90011-7

Hajcak, G., Holroyd, C. B., Moser, J. S., \& Simons, R. F. (2005). Brain potentials associated with expected and unexpected good and bad outcomes. Psychophysiology, 42(2), 161-170. doi:https://doi.org/10. $1111 / \mathrm{j} .1469-8986.2005 .00278 . \mathrm{x}$
Hajcak, G., Moser, J. S., Holroyd, C. B., \& Simons, R. F. (2007). It's worse than you thought: The feedback negativity and violations of reward prediction in gambling tasks. Psychophysiology, 44(6), 905912. doi:https://doi.org/10.1111/j.1469-8986.2007.00567.x

Hinojosa, J., Mercado, F., \& Carretié, L. (2015). N170 sensitivity to facial expression: a meta-analysis. Neuroscience \& Biobehavioral Reviews, 55, 498-509. doi:https://doi.org/10.1016/j.neubiorev. 2015.06.002

Holroyd, C. B., \& Coles, M. G. H. (2002). The neural basis of human error processing: reinforcement learning, dopamine, and the errorrelated negativity. Psychological Review, 109(4), 679-709. doi: https://doi.org/10.1037/0033-295X.109.4.679

Holroyd, C. B., Pakzad-Vaezi, K. L., \& Krigolson, O. E. (2008). The feedback correct-related positivity: sensitivity of the event-related brain potential to unexpected positive feedback. Psychophysiology, 45(5), 688-697. doi:https://doi.org/10.1111/j.1469-8986.2008. 00668.x

Huang, Y., \& Yu, R. (2014). The feedback-related negativity reflects "more or less" prediction error in appetitive and aversive conditions. Frontiers in Neuroscience, 8, 108. doi:https://doi.org/10.3389/fnins. 2014.00108

Ito, T. A., Larsen, J. T., Smith, N. K., \& Cacioppo, J. T. (1998). Negative information weighs more heavily on the brain: The negativity bias in evaluative categorizations. Journal of Personality and Social Psychology, 75(4), 887. doi:https://doi.org/10.1037//0022-3514.75. 4.887

Johnson, R. (1988). The amplitude of the P300 component of the eventrelated potential: review and synthesis. In P. Ackles, J.R. Jennings, \& M. G. H. Coles (Eds.), Advances in Psychophysiology (Vol. 3, pp. 69-137). Greenwich, CT: JAI Press.

Johnson, R., \& Donchin, E. (1985). Second thoughts: Multiple P300s elicited by a single stimulus. Psychophysiology, 22(2), 182-194. doi:https://doi.org/10.1111/j.1469-8986.1985.tb01584.x

Johnston, P., Molyneux, R., \& Young, A. W. (2014). The N170 observed 'in the wild': robust event-related potentials to faces in cluttered dynamic visual scenes. Social Cognitive and Affective Neuroscience, 10(7), 938-944. doi:https://doi.org/10.1093/scan/ nsu136

Kayser, J., Tenke, C. E., Kroppmann, C. J., Fekri, S., Alschuler, D. M., Gates, N. A., . . . Bruder, G. E. (2010). Current source density (CSD) old/new effects during recognition memory for words and faces in schizophrenia and in healthy adults. International Journal of Psychophysiology, 75(2), 194-210. doi:https://doi.org/10.1016/j. ijpsycho.2009.12.001

Langner, O., Dotsch, R., Bijlstra, G., Wigboldus, D. H. J., Hawk, S. T., \& van Knippenberg, A. (2010). Presentation and validation of the Radboud Faces Database. Cognition \& Emotion, 24(8), 13771388. doi:https://doi.org/10.1080/02699930903485076

Levine, D. K. (1998). Modeling Altruism and Spitefulness in Experiments. Review of Economic Dynamics, 1(3), 593-622. doi: https://doi.org/10.1006/redy.1998.0023

Ma, Q., Hu, Y., Jiang, S., \& Meng, L. (2015). The undermining effect of facial attractiveness on brain responses to fairness in the Ultimatum Game: an ERP study. Frontiers in Neuroscience, 9, 77. doi:https:// doi.org/10.3389/fnins.2015.00077

Maier, M. E., \& Steinhauser, M. (2013). Updating expected action outcome in the medial frontal cortex involves an evaluation of error type. Journal of Neuroscience, 33(40), 15705-15709. doi:https:// doi.org/10.1523/JNEUROSCI.2785-13.2013

Milne, E. (2011). Increased intra-participant variability in children with autistic spectrum disorders: evidence from single-trial analysis of evoked EEG. Frontiers in Psychology, 2, 51. doi:https://doi.org/ 10.3389/fpsyg.2011.00051

Miltner, W. H., Braun, C. H., \& Coles, M. G. (1997). Event-related brain potentials following incorrect feedback in a time-estimation task: evidence for a "generic" neural system for error detection. Journal 
of Cognitive Neuroscience, 9(6), 788-798. doi:https://doi.org/10. 1162/jocn.1997.9.6.788

Mognon, A., Jovicich, J., Bruzzone, L., \& Buiatti, M. (2011). ADJUST: An automatic EEG artifact detector based on the joint use of spatial and temporal features. Psychophysiology, 48(2), 229-240. doi: https://doi.org/10.1111/j.1469-8986.2010.01061.x

Morgenstern, O., \& Von Neumann, J. (1953). Theory of games and economic behavior: Princeton University Press.

Mussel, P., Hewig, J., Allen, J. J., Coles, M. G., \& Miltner, W. (2014). Smiling faces, sometimes they don't tell the truth: Facial expression in the ultimatum game impacts decision making and event-related potentials. Psychophysiology, 51(4), 358-363. doi:https://doi.org/10. 1111/psyp. 12184

Mussel, P., Hewig, J., \& Weiß, M. (2018). The reward-like nature of social cues that indicate successful altruistic punishment. Psychophysiology, 55(9), e13093. doi:https://doi.org/10.1111/psyp. 13093

Niedenthal, P. M., Mermillod, M., Maringer, M., \& Hess, U. (2010). The Simulation of Smiles (SIMS) model: Embodied simulation and the meaning of facial expression. Behavioral and Brain Sciences, 33(6), 417. doi:https://doi.org/10.1017/S0140525X10000865

Osinsky, R., Mussel, P., Ohrlein, L., \& Hewig, J. (2014). A neural signature of the creation of social evaluation. Social Cognitive and Affective Neuroscience, 9(6), 731-736. doi:https://doi.org/10.1093/ scan $/ \mathrm{nst} 051$

Peirce, J. W. (2008). Generating Stimuli for Neuroscience Using PsychoPy. Frontiers in Neuroinformatics, 2, 10. doi:https://doi.org/ 10.3389/neuro.11.010.2008

Pfabigan, D. M., Alexopoulos, J., Bauer, H., Lamm, C., \& Sailer, U. (2011). All about the money-external performance monitoring is affected by monetary, but not by socially conveyed feedback cues in more antisocial individuals. Frontiers in Human Neuroscience, 5, 100. doi:https://doi.org/10.3389/fnhum.2011.00100

Polich, J. (2007). Updating P300: an integrative theory of P3a and P3b. Clinical Neurophysiology, 118(10), 2128-2148. doi:https://doi.org/ 10.1016/j.clinph.2007.04.019

Qu, C., Wang, Y., \& Huang, Y. (2013). Social exclusion modulates fairness consideration in the ultimatum game: an ERP study. Frontiers in Human Neuroscience, 7, 505. doi:https://doi.org/10.3389/fnhum. 2013.00505

Rossion, B., Gauthier, I., Tarr, M. J., Despland, P., Bruyer, R., Linotte, S., \& Crommelinck, M. (2000). The N170 occipito-temporal component is delayed and enhanced to inverted faces but not to inverted objects: an electrophysiological account of face-specific processes in the human brain. Neuroreport, 11(1), 69-72. doi:https://doi.org/10. 1097/00001756-200001170-00014

Sambeth, A., Maes, J., \& Brankačk, J. (2004). With long intervals, interstimulus interval is the critical determinant of the human P300 amplitude. Neuroscience Letters, 359(3), 143-146. doi:https://doi.org/ 10.1016/j.neulet.2004.01.064

Schreiner, T., Alexopoulos, J., Pfabigan, D., \& Sailer, U. (2010). Facial cues affect the feedback negativity to offers in the Ultimatum Game. An EEG investigation. International Journal of Psychophysiology, 77(3), 337. doi:https://doi.org/10.1016/j.ijpsycho.2010.06.275

Schupp, H. T., Markus, J., Weike, A. I., \& Hamm, A. O. (2003). Emotional facilitation of sensory processing in the visual cortex. Psychological Science, 14(1), 7-13. doi:https://doi.org/10.1111/ 1467-9280.01411

Sharot, T. (2011). The optimism bias. Current biology, 21(23), R941R945. doi:j.cub.2011.10.030

Weiß, M., Gutzeit, J., Rodrigues, J., Mussel, P., \& Hewig, J. (2019a). Do emojis influence social interactions? Neural and behavioral responses to affective emojis in bargaining situations. Psychophysiology, 56(4), e13321. doi:https://doi.org/10.1111/psyp. 13321

Weiß, M., Mussel, P., \& Hewig, J. (2019b). The value of a real face: Differences between affective faces and emojis in neural processing and their social influence on decision-making. Social Neuroscience. doi:https://doi.org/10.1080/17470919.2019.1675758

Winkler, I., Haufe, S., \& Tangermann, M. (2011). Automatic classification of artifactual ICA-components for artifact removal in EEG signals. Behavioral and Brain Functions, 7(1), 30. doi:https://oi.org/ 10.1186/1744-9081-7-30

Wu, Y., Hu, J., van Dijk, E., Leliveld, M. C., \& Zhou, X. (2012). Brain activity in fairness consideration during asset distribution: does the initial ownership play a role? PLOS ONE, 7(6), e39627. doi:https:// doi.org/10.1371/journal.pone.0039627

Zaki, J., Kallman, S., Wimmer, G. E., Ochsner, K., \& Shohamy, D. (2016). Social cognition as reinforcement learning: Feedback modulates emotion inference. Journal of Cognitive Neuroscience, 28(9), 1270-1282. doi:https://doi.org/10.1162/jocn_a_00978

Publisher's note Springer Nature remains neutral with regard to jurisdictional claims in published maps and institutional affiliations. 